\title{
Reconstructing rare soil microbial genomes using in situ enrichments and metagenomics
}

\author{
Tom O. Delmont 1,2*, A. Murat Eren ${ }^{2}$, Lorrie Maccario ${ }^{1}$, Emmanuel Prestat ${ }^{1}$, \\ Özcan C. Esen ${ }^{2}$, Eric Pelletier ${ }^{3,4,5}$, Denis Le Paslier ${ }^{3,4,5}$, Pascal Simonet ${ }^{1}$ and \\ Timothy M. Vogel ${ }^{1}$
}

\begin{abstract}
${ }^{1}$ Environmental Microbial Genomics, Laboratoire Ampere, Centre National de la Recherche Scientifique, Ecole Centrale de Lyon, Université de Lyon, Ecully, France, ${ }^{2}$ Josephine Bay Paul Center for Comparative Molecular Biology and Evolution, Marine Biological Laboratory, Woods Hole, MA, USA, ${ }^{3}$ Commissariat à l'Energie Atomique et aux Energies Alternatives, Genoscope, Evry, France, ${ }^{4}$ UMR8030, Centre National de la Recherche Scientifique, Evry, France, ${ }^{5}$ Université d'Evry Val d'Essonne, Evry, France
\end{abstract}

\section{OPEN ACCESS}

Edited by:

Jorge L. M. Rodrigues,

University of California, Davis, USA

Reviewed by:

Kristen M. DeAngelis,

University of Massachusetts Amherst,

Peter Bergholz,

USA

North Dakota State University, USA

*Correspondence:

Tom O. Delmont,

Josephine Bay Paul Center for

Comparative Molecular Biology and

Evolution, Marine Biological

Laboratory, 7 MBL Street, Woods

Hole, MA 02543, USA

tdelmont@mbl.edu

Specialty section:

This article was submitted to

Systems Microbiology,

a section of the journal

Frontiers in Microbiology

Received: 01 March 2015

Accepted: 09 April 2015

Published: 30 April 2015

Citation:

Delmont TO, Eren AM, Maccario L, Prestat E, Esen ÖC, Pelletier E, Le Paslier $D$, Simonet $P$ and Vogel TM

(2015) Reconstructing rare soil microbial genomes using in situ enrichments and metagenomics.

Front. Microbiol. 6:358

doi: 10.3389/fmicb.2015.00358
Despite extensive direct sequencing efforts and advanced analytical tools, reconstructing microbial genomes from soil using metagenomics have been challenging due to the tremendous diversity and relatively uniform distribution of genomes found in this system. Here we used enrichment techniques in an attempt to decrease the complexity of a soil microbiome prior to sequencing by submitting it to a range of physical and chemical stresses in 23 separate microcosms for 4 months. The metagenomic analysis of these microcosms at the end of the treatment yielded $540 \mathrm{Mb}$ of assembly using standard de novo assembly techniques (a total of 559,555 genes and 29,176 functions), from which we could recover novel bacterial genomes, plasmids and phages. The recovered genomes belonged to Leifsonia $(n=2)$, Rhodanobacter $(n=5)$, Acidobacteria $(n=2)$, Sporolactobacillus ( $n=2$, novel nitrogen fixing taxon), Ktedonobacter $(n=1$, second representative of the family Ktedonobacteraceae), Streptomyces $(n=3$, novel polyketide synthase modules), and Burkholderia ( $n=2$, includes mega-plasmids conferring mercury resistance). Assembled genomes averaged to $5.9 \mathrm{Mb}$, with relative abundances ranging from rare $(<0.0001 \%)$ to relatively abundant $(>0.01 \%)$ in the original soil microbiome. Furthermore, we detected them in samples collected from geographically distant locations, particularly more in temperate soils compared to samples originating from high-latitude soils and deserts. To the best of our knowledge, this study is the first successful attempt to assemble multiple bacterial genomes directly from a soil sample. Our findings demonstrate that developing pertinent enrichment conditions can stimulate environmental genomic discoveries that would have been impossible to achieve with canonical approaches that focus solely upon post-sequencing data treatment.

Keywords: rare biosphere, soil, metagenomics, environmental genomics, plasmids, phages

\section{Introduction}

Soil microbial communities might display the highest level of bacterial diversity of any environment with a single gram reported to contain about a billion cells making up thousands to millions of different taxa (Torsvik et al., 2002; Gans et al., 2005; Roesch et al., 2007). 
These microorganisms harbor a large portion of Earth's biomass and are responsible for a range of critical functions including those that affect climate (Falkowski et al., 2000; Finzi et al., 2011), agriculture production (Kennedy and Smith, 1995; Giller et al., 1997), bioremediation (Galvão et al., 2005; Boubakri et al., 2006), pharmaceutical and other industrial applications (Malpartida and Hopwood, 1984; Daniel, 2004; Jacquiod et al., 2013). However, despite advances in both data generation and analysis methods during the last decade, genomic recovery from soil still represents the most critical bottleneck to develop an understanding of the evolutionary history, range of adaptation and functions of soil microorganisms.

Metagenomic approaches (the analysis of DNA recovered from the environment) have been developed to by-pass the limitations associated with cultivation efforts (Stahl et al., 1984; Handelsman et al., 1998; Rondon et al., 2000) and are now widely used to estimate the structure and functional potential of microorganisms found in soil samples (Tringe et al., 2005; Delmont et al., 2011b, 2012; Mackelprang et al., 2011; Fierer et al., 2012; Jacquiod et al., 2013; David et al., 2014; Nesme et al., 2014). Today, most soil metagenomic studies rely on taxonomic or functional annotation of short reads based on curated databases. However, the limited sensitivity of short reads restrains the fuller explanation of the available sequencing information (Wommack et al., 2008; Delmont et al., 2011a).

A more biologically promising yet computationally challenging alternative is to assemble short reads back together to reconstruct genomes and other genetic structures in a de novo manner. This approach was successfully conducted in various environments, including acid mine drainage biofilms (Tyson et al., 2004), sludges (Garcia Martin et al., 2006; Albertsen et al., 2013), human feces (Sharon et al., 2013), cow rumen (Hess et al., 2011), permafrost soil (Mackelprang et al., 2011), hypersaline lakes (Narasingarao et al., 2012), hot spring microbial mat (Liu et al., 2012) and hydrothermal plumes (Anantharaman et al., 2013). However, the genetic distance between the different members of the ecosystem affects the efficiency of the assembly process (Luo et al., 2012; Mende et al., 2012; Albertsen et al., 2013; Nielsen et al., 2014), leading to a drastic decline of recovery in metagenomic assemblies of complex communities.

Soil samples harbor large numbers of uniformly distributed microbial genomes, and approaches that work for other environments fail to recover genomes from soil in a systematical manner as they quickly hit the computational limitations of assembly algorithms. As a consequence, recovering even the most abundant genomes from temperate soils through metagenomic assembly approaches have yet to be accomplished (Delmont et al., 2012; Pell et al., 2012; Howe et al., 2014). For instance, Howe et al. (2014) generated 1.8 and 3.3 billion reads from two soil metagenomes, yet the length of genetic structures they could assemble remained below $21 \mathrm{~kb}$ and $3 \mathrm{~kb}$, respectively. Today, the challenge to recover large genetic structures from a soil metagenome is unlikely to be addressed with deeper sequencing. However, employing biologically relevant modifications that take place before sequencing can reduce the complexity of challenges that arise after sequencing. For instance, we hypothesize that altering the structure of a soil microbiome in order to reduce its complexity by favoring the emergence of dominant microbial populations can improve metagenomic assemblies.

Microcosm enrichment is a common approach in bioremediation (i.e., employing microbes to degrade toxic chemical compounds) and bioprospecting (Wagner-Döbler et al., 1998; Ibekwe et al., 2001; Yakimov et al., 2005; McKew et al., 2007). Enrichment studies were also used in combination with metagenomics to recover new functions from soil (Jacquiod et al., 2013; David et al., 2014; Delmont et al., 2014) and successfully recovered novel genomes from less diverse environments (McIlroy et al., 2013). Here, we implement an enrichment-based "divide and conquer" approach in an attempt to reconstruct novel bacterial genomes directly from a soil sample collected from the Long-term Park Grass experiment, Rothamsted, UK, where previous efforts to reconstruct genomes using direct sequencing approaches failed (Delmont et al., 2012). We divided our soil sample into 23 identical microcosms and subjected them to various environmental stress conditions (ESCs), including mercury, ethanol, and diesel enrichments, in an attempt to stimulate different parts of the microbiome. After 4 months of treatment, we extracted, sequenced and assembled genetic material from these microcosms.

\section{Material and Methods}

\section{Sampling}

A soil sample from the top 21 centimeters of the ground was collected in July 2010 from the Park Grass experiment (Vogel et al., 2009), Rothamsted Research, Hertfordshire, UK, using sterile manual corers $(10 \mathrm{~cm}$ diameter $)$ and was placed in sterile plastic bags, sealed and placed at room temperature $24 \mathrm{~h}$ while it was transported to Lyon, France. The sample was then sieved $(2 \mathrm{~mm})$ and directly used for the microcosm experiment.

\section{Microcosm Conditions}

Microcosms were done in triplicates (50 $\mathrm{g}$ of soil in each microcosm), stored at room temperature (except for the high temperature condition), without light, and were hermetically closed during the experiment. Following ESCs applied to each microcosm: ESC C (control): $5 \mathrm{ml}$ of purified water was sprayed for control; ESC 1: $5 \mathrm{ml}$ of purified water with ethanol $(20 \%$ of the volume) was sprayed for ethanol enrichment; ESC 2: $5 \mathrm{ml}$ of purified water with $\mathrm{NaCl}(30 \mathrm{~g} / \mathrm{L})$ was sprayed for salt enrichment \#1; ESC 3: $5 \mathrm{ml}$ of purified water with $\mathrm{NaCl}(300$ $\mathrm{g} / \mathrm{L}$, salt saturation) was sprayed for salt enrichment \#2; ESC 4: $5 \mathrm{ml}$ of purified water was sprayed and then microcosms were incubated at $37^{\circ} \mathrm{C}$ for high temperature condition; ESC 5: $5 \mathrm{ml}$ of purified water was sprayed and then, the microcosm atmosphere was replaced by nitrogen gas for nitrogen atmospheric condition; ESC 6: $5 \mathrm{ml}$ of purified water with diesel (for a final concentration of $50 \mathrm{~g} / \mathrm{kg}$ of soil) was sprayed for diesel enrichment; ESC 7: $5 \mathrm{ml}$ of purified water with heavy metals (zinc, cadmium, nickel, and cobalt, for a final concentration of $0.2 \mathrm{~g} / \mathrm{kg}$ of soil for each metal) was sprayed for heavy metals enrichment \#1; ESC 8: $5 \mathrm{ml}$ of purified water with heavy metals (zinc, cadmium, nickel, and cobalt, for a final concentration of $2 \mathrm{~g} / \mathrm{kg}$ of soil for each metal) was sprayed for heavy metals enrichment \#2; ESC 
9: $5 \mathrm{ml}$ of purified water with inorganic mercury $\left(\mathrm{HgCl}_{2}\right.$ for a final concentration of $0.02 \mathrm{~g} / \mathrm{kg}$ of soil) was sprayed for mercury enrichment \#1; ESC 10: $5 \mathrm{ml}$ of purified water with inorganic mercury $\left(\mathrm{HgCl}_{2}\right.$ for a final concentration of $0.2 \mathrm{~g} / \mathrm{kg}$ of soil for each metal) was sprayed for mercury enrichment \#2. Note that microcosms were aerated (by opening the cap for about a minute) every week to renewing the normal air condition. For the nitrogen condition (ESC 5), this step was immediately followed by replacing the air with nitrogen gas.

\section{DNA Extraction, Quantification, and Fingerprint Analyzes}

After 4 months of incubation, DNA samples were extracted from $0.5 \mathrm{~g}$ of soil using the MP BIO $1 \mathrm{O} 1$ fast prep (Biomedical, Eschwege, Germany), a protocol known to be relatively efficient for this particular soil (Delmont et al., 2011b). Samples were purified using GFX columns (GE Healthcare) (final volume of 40 microliters) and the DNA was finally quantified using the Qubit ${ }^{\circledR}(1.0)$ Fluorometer. A minimum of six DNA extractions were used to estimate the quantity of DNA extracted and purified from each microcosm. Ribosomal intergenic spacer analyzes (RISA) were done for each microcosm to study condition reproducibility in term of microbial community relative composition. See (Delmont et al., 2011b) for more details about RISA analyses. RISA profiles were reproducible for all ESCs but the mercury enrichment \#2 were an outlier microcosm was detected (Figure S1).

\section{Metagenomic Sequencing}

A minimum of $10 \mu \mathrm{g}$ of DNA were used to generate metagenomic libraries for each Roche/454 pyrosequencing run on a 454 pyrosequencer (GS FLX Titanium Series Reagents; Roche 454; Shirley, NY, USA). In a first phase, pair-end sequencing was done on duplicate microcosms of each ESC (about one million reads per microcosm). As an exception, the three replicates of the mercury enrichment $\# 2$ were sequenced due to a low reproducibility of one microcosm observed using RISA profiles. Thus, 23 metagenomic data were generated. In a second phase, a mate-pair sequencing effort with $3 \mathrm{~kb}$ of gap was done for duplicates corresponding to 3 promising ESCs: heavy metals enrichment \#2 (one million reads each), mercury enrichment \#1 (two million reads each) and the outlier replicate of the mercury enrichment \#2 (one million reads). Duplicates corresponding to the ethanol enrichment were also further sequenced, however, due to the high fragmentation of the DNA recovered from this ESC, additional sequencing was done with paired-end library preparation instead of mate-pair.

\section{Data Analyses}

Raw metagenomic reads were annotated using MG-RAST (Meyer et al., 2008). Detected functions and taxa were normalized to $100 \%$ in each sample, and a Kruskal-Wallis test was performed for Pfams and genera using the $\mathrm{R}$ package vegan (Oksanen et al., 2007). Metagenomic data were also sequentially assembled (Newbler software v.05, assembly requirement of $96 \%$ identity over a minimum length of $100 \mathrm{nt}$, with reads limited to one contig/scaffold). Coverage, GC-content and tetranucleotide frequency information was used to bin contigs/scaffold into draft genomes. Visualization of this information was done using in-house Python programs. Genomes and other genetic structures reconstructed were subsequently annotated using RAST (Aziz et al., 2008) and visualized using Artemis and DNAPlotter (Carver et al., 2009). Completions estimates were done using a collection of single-copy genes ( $\mathrm{Wu}$ and Eisen, 2008). Metagenomic datasets were then mapped to these genetic structures (using CLC v.6 and a mapping requirement of $97 \%$ and $90 \%$ identity over the full read length) to estimate their distribution in the different microcosms as well as in the natural microbial community (Delmont et al., 2012) and in communities from distant soil biomes (Fierer et al., 2012). Positive detection was defined when a minimum of 10 reads from the targeted structure were detected in the selected dataset. Gephi v0.8.2 (Bastian et al., 2009) was used to generate functional networks (Force Atlas 2) connecting different collections of reconstructed genomes and an exhaustive list of genomes affiliated to the same taxonomy available in NCBI in April 2014: 24 Streptomyces, 18 Chloroflexi, 10 Acidobacteria, 6 Rhodanobacter, and 3 Sporolactobacillus. In parallel, sequences related to the $16 \mathrm{~S}$ rRNA gene were screened from metagenomic datasets using RDP (Cole et al., 2009) database through MG-RAST (Meyer et al., 2008) with a minimum of $200 \mathrm{nt}$ of alignment and $90 \%$ identity. Sequences so collected were partitioned by genus and independently assembled on Newbler (100 nt alignment, 99\% identity minimum, reads limited to one contig) to create consensus sequences. The metagenomic dataset is publically available at http://www.genomenviron.org/Projects/METASOIL. html. Draft genomes are publically available at http://dx.doi. org/10.6084/m9.figshare.1320632 and in the NCBI Bioproject PRJNA279807.

\section{S rRNA Gene Amplicons}

A 16S rRNA gene amplification step was done for DNA pools extracted from the seven microcosms targeted during the second sequencing phase as well as one microcosm representing the control condition. Primers pA ( $5^{\prime}$ AGA GTT TGA TCC TGG CTC AG $3^{\prime}$ ) and $\mathrm{pH}\left(5^{\prime}\right.$ AAG GAG GTG ATC CAG CCG CA $\left.3^{\prime}\right)$ were used for this amplification. PCR products were then gel-purified (GE Healthcare illustra GFX PCR DNA and Gel Band Purification Kit) and cloned in a TOPO-TA using the TOPO-TA cloning kit for Sequencing (Invitrogen). 96 clones for each microcosm condition were randomly selected and inoculated in a 96 well plate. After TOPO-TA vector extraction, inserts were sequenced with Sanger Technology (Beckmann Coulter Genomics). Both primer M13for ( $5^{\prime}$ TGT AAA ACG ACG GCC AGT 3) and primer M13rev (5' CAG GAA ACA GCT ATG ACC $3^{\prime}$ ) reactions were done. Sequences obtained with forward and reverse reactions were assembled and vectorpurified with Seqman Software (Lasergen Seqman DNASTAR). Consensus sequences were then compiled by dataset for further data analysis.

\section{Taxonomical Inference}

Genomic taxonomical affiliation was inferred using information extracted from (i) global sequence similarity with the RAST 
collection of genomes (Aziz et al., 2008), (ii) full or fragmented $16 \mathrm{~S}$ rRNA genes present in assembled genomes, (iii) sequences related to $16 \mathrm{~S}$ rRNA gene assembled after their screening at the genus level in unassembled metagenomic datasets, and (iv) $16 \mathrm{~S}$ rRNA gene amplicon datasets.

\section{Results and Discussion}

\section{ESCs Stimulate the Reconstruction of Genetic Material from the Soil Microbiome}

We sequenced the genetic material of two microcosms for each ESC as well as the outlier microcosm replicate of the mercury enrichment \#2 (see Figure S1), thus generating 23 metagenomic datasets. 1.05 ( \pm 0.17) million pyrosequencing reads of about 350 nucleotides were generated for each microcosm, representing a total of 24.05 million reads. We combined the 23 unassembled metagenomic dataset with the 13 dataset previously generated from the natural community of the same site for community composition comparison purposes (Delmont et al., 2012). The latter dataset was generated with the same sequencing strategy and provides background regarding the natural and methodological fluctuation of the Park Grass soil microbiome. MG-RAST identified 4,081 genera and 8,541 distinct protein families (Pfams) from the combined datasets (Tables S1, S2). Among these, several taxonomical groups and Pfams varied significantly between different conditions (Kruskal-Wallis test, $p$ value $<0.05)$, indicating that the microcosm strategy initiated a shift in the composition of the initial microbial community (Figure 1). Overall, the proportion of Alphaproteobacteria decreased in all ESCs, which benefited various ESC-specific taxa (e.g., Ktedonobacter, Bacilli, Betaproteobacteria). We detected positive selective pressure effect on functions directly related to ESCs: the Pfam related to Cobalt-zinc-cadmium resistance protein $\mathrm{CzcD}$ was more prevalent under the heavy metal enrichment \#2, anaerobic cytochrome c552 was higher in soil under nitrogen gas atmosphere, and mercury resistance operon regulatory protein was higher in the mercury enrichments. Moreover, some functions were reproducibly detected only under specific conditions, such as IncIl plasmid conjugative transfer DNA primase in microcosms from the heavy metal enrichment \#2.

For most ESCs, we observed a decrease in the total amount of DNA, suggesting overall population declines throughout the incubation period (Figure S2). DNA extraction yield varied from $14.6( \pm 2.7) \mu \mathrm{g}$ to $0.7( \pm 0.4) \mu \mathrm{g}$ per gram of soil depending on the ESC while it was about $25 \mu \mathrm{g}$ for the untreated sample. However, the taxonomical and functional shifts indicated that the ESCs impacted different parts of the soil microbiome, providing access to distinct genomic populations through assembly. We assembled each metagenome obtained from microcosms separately, and RAST annotation identified a total of 386,684 genes (average length of $451 \mathrm{nt}$ ) in contigs ranging from 0.1 to $300 \mathrm{~kb}$, with $59 \%$ of them longer than $1 \mathrm{~kb}$. Assembly efficiency and average length of genes were substantially enhanced for many of the ESCs in comparison to our controls (Figures 2A-D). Interestingly, although some conditions, such as Nitrogen headspace, provided a different community structure, they did not enhance the assembly, suggesting that a large number of microbial populations were co-selected. $52.4 \%$ of the genes were successfully annotated in RAST (Table S3) and affiliated to 24,291 different functions, most of which were related to Bacteria. We detected three times more functions through $\sim 400$ thousand assembled genes

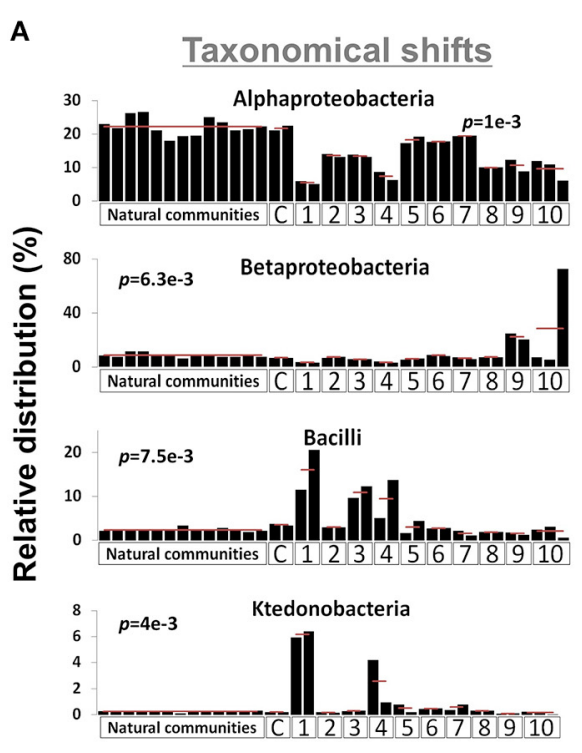

FIGURE 1 | Graphs represent the relative distribution of 4 bacterial taxa using M5NR databases $(A)$ and Pfams $(B)$ in the 36 metagenomic datasets when annotated in MG-RAST. $E$-value cut-off was defined as 10-5. P-values were defined using distribution

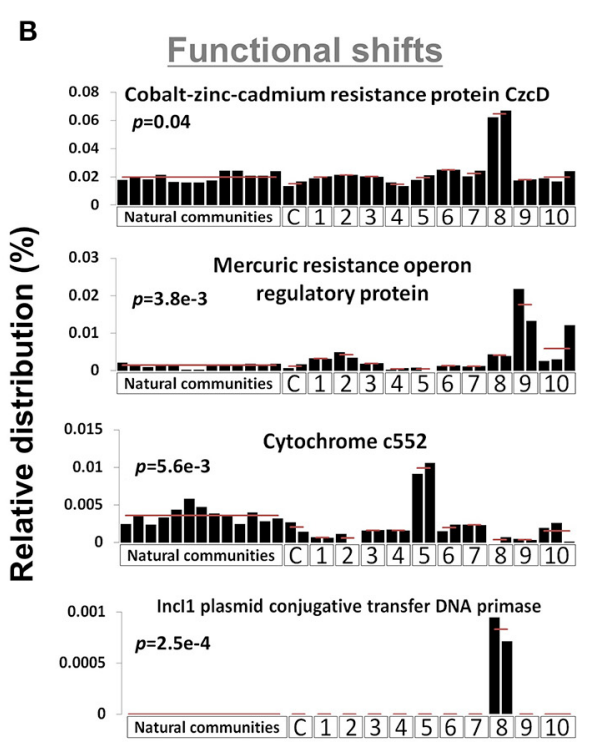

variations between conditions (Kruskal-Wallis test). X-axes identify different ESCs: C, control; 1, ethanol; 2, salt \#1; 3, salt \#2; 4, 37 ${ }^{\circ}$; 5 , nitrogen; 6, diesel; 7 , heavy metals \#1; 8 , heavy metals \#2; 9, mercury \#1; 10, mercury \#2. 


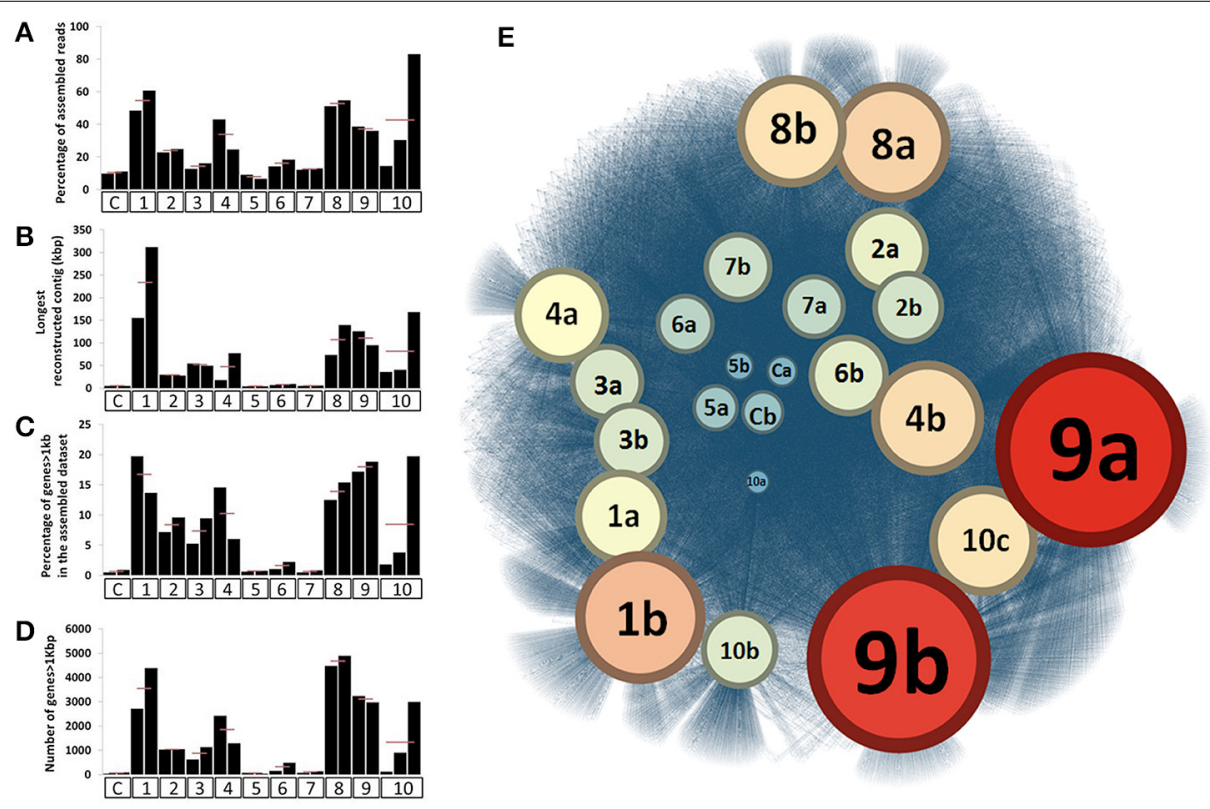

FIGURE 2 | Panels (A,B) represent the relative percentage of assembled reads and longest reconstructed contig after assembling the $\mathbf{2 3}$ datasets, respectively. Panels (C,D) represent the number and percentage of genes longer than $1 \mathrm{~kb}$ recovered from these assemblies, respectively. For (A-D), X-axes identify different ESCs. Finally, (E) represents a functional network linking the 23 assembled metagenomic datasets and the 24,291 distinct functions annotated from these assemblies. The network was generated using Gephi and Force Atlas 2 and represents a total of 85,188 connections. Datasets are colored and have a size depending on the number of different functions they are connected to. In all panels, ESCs represent the following conditions; C, control; 1 , ethanol; 2, salt \#1; 3 , salt \#2; $4,37^{\circ} \mathrm{C} ; 5$, nitrogen; 6 , diesel; 7 , heavy metals \#1; 8 , heavy metals \#2; 9, mercury \#1; 10, mercury \#2. with RAST, compared to $\sim 36$ million unassembled reads using MG-RAST. This functional recovery represents a significant achievement in comparison to what was previously detected from the direct sequencing effort of the Park Grass microbial community (Delmont et al., 2012).

Our network analysis connecting 24,291 functions to microcosms they originate demonstrated the efficacy of multiple ESCs to stimulate the recovery of different functional pools (Figure 2E). In particular, the mercury enrichment \#1 provided a total of 3,155 unique functions from 68,450 assembled genes, representing an 18-fold increase compared to controls (176 unique functions only). Overall, this analysis showed that ethanol, heavy metals and mercury ESCs resulted in highest rates of gene and function recovery through metagenomic assemblies.

\section{We Assembled $1 \%$ of the Soil Microbiome and Recovered Novel Bacterial Genomes}

We performed additional sequencing for seven microcosms that showed highest potential for recovery of genomes (ethanol enrichment, heavy metals enrichment \#2 and mercury enrichments \#1 and \#2) based on metagenomic assembly scores (Figure 2). Assembly of the additional data resulted in genetic structures up to $4 \mathrm{Mb}$ and lead to a total recovery of 334,576 coding genes and 3,820 RNAs from these seven microcosms using about $6 \mathrm{~Gb}$ of sequencing. Overall, we recovered 540 $\mathrm{Mb}$ of genetic material that harbors 559,555 genes (average length of $466 \mathrm{nt}$, includes 56,228 genes longer than 1,000 nt) representing 29,176 functions. This genetic material constitutes $1-2.5 \%$ of this soil microbiome at $97-90 \%$ identity cut-off, respectively. Ninety-seven percent identity cut-off complies with strain variation and sequencing errors and was selected for downstream analysis of the Park Grass soil microbiome. We used a tetranucleotide frequency-based supervised binning approach to identify draft genomes using contigs and scaffolds longer than $10 \mathrm{~kb}$ (Figure 3). In most cases, clusters displayed stable coverage and GC-content scores. Contigs that make up draft genomes of similar taxonomical affiliation most of the time organized in the same tetranucleotide frequency cluster, or showed consistent coverage when they were split into multiple clusters (Figure 3).

We determined a total of $17 \mathrm{draft}$ genomes from these microcosms. Table 1 reports the functional properties and taxonomical affiliations of these genomes, which represent Leifsonia $(n=2)$, Rhodanobacter $(n=5)$, Acidobacteria $(n=2)$, Sporolactobacillus $(n=2)$, Ktedonobacter $(n=$ $1)$, Streptomyces $(n=3)$, and Burkholderia $(n=2)$. They carry between 2,637 and 9,051 genes and possess a global GCcontent ranging from $47.3 \%$ to $71 \%$. Our analysis of singlecopy gene families revealed that 11 of 17 bacterial genomes with no close relatives in their microcosms were $97 \%$ complete in average (Table 1). In contrast, the same analysis for the remaining $6 \mathrm{draft}$ genomes that co-occur with their close relatives in microcosms they were found (i.e., two Streptomyces genomes and the two Burkholderia genomes) estimated that they were $61 \%$ complete in average, despite their large sizes (up to $9.3 \mathrm{Mb}$ 


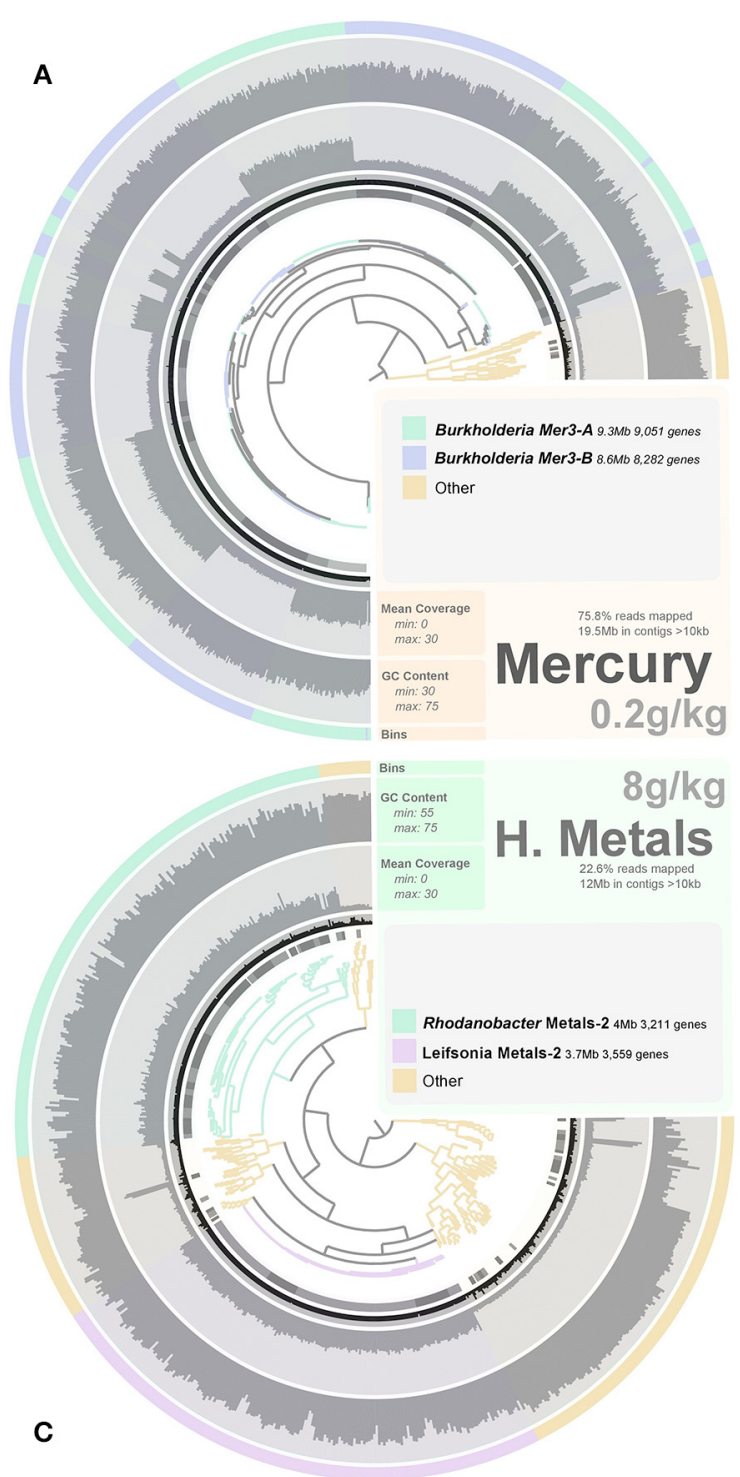

FIGURE 3 | Panels (A-D) exhibit the metagenomic assemblies (genetic structures reaching $\mathbf{1 0} \mathrm{kb}$ only) recovered from the Mercury enrichments $2(19.5 \mathrm{Mb})$ and 1 (33.8 Mb), heavy metals enrichment 2 (12 Mb) and ethanol enrichment (10 Mb), respectively. We applied a mapping requirement of $97 \%$ identity to estimate coverage values. Genetic structures are organized in trees based on their tetranucleotide frequency (Euclidean distance) and were subsequently fragmented into sections of

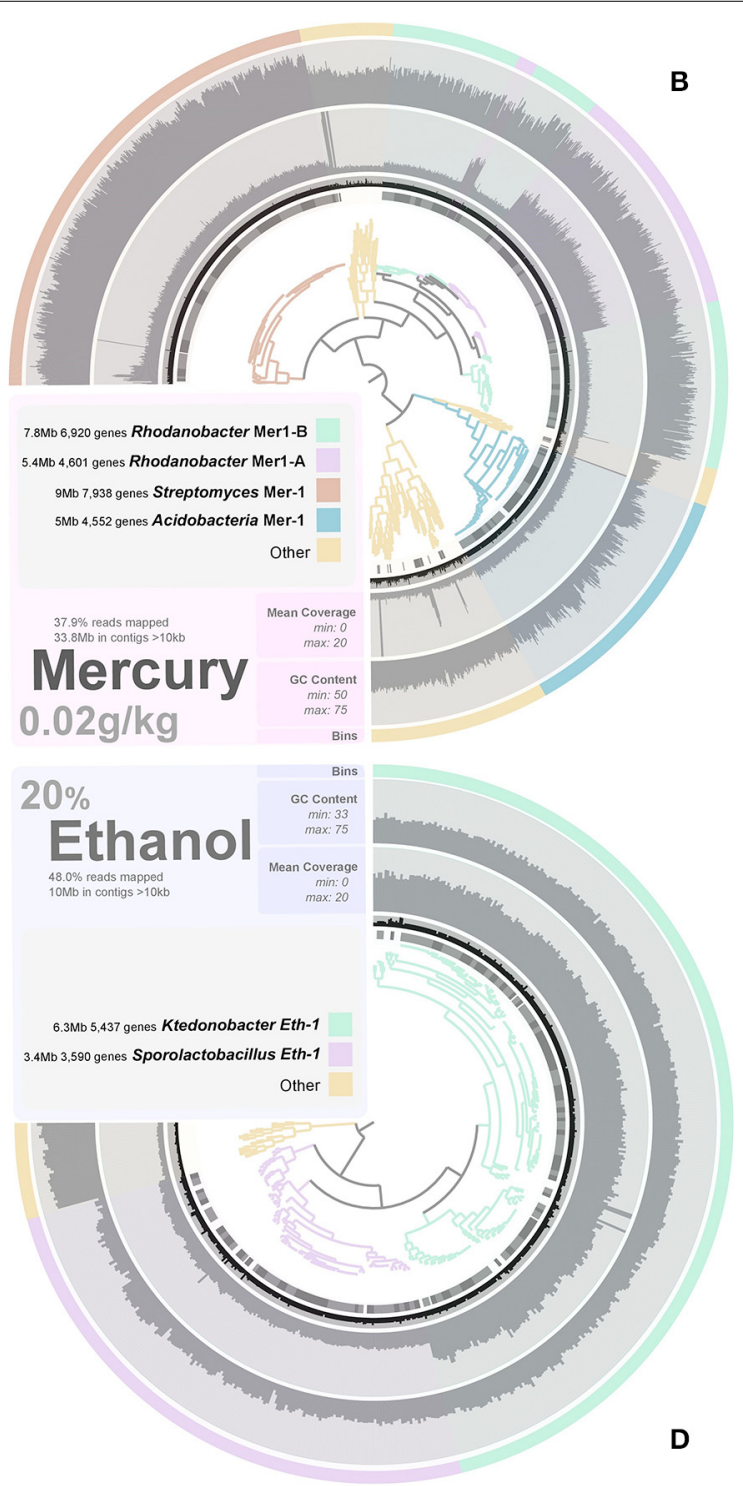

$20 \mathrm{~kb}$ displaying the same color in the first outer cycle. Therefore, each section in the tree represents a genetic structure ranging from 10 to $20 \mathrm{~kb}$ (length is displayed in the second outer cycle in black). Mean coverage (third outer cycle) and GC-content (forth outer cycle) are display for each section to assess the coherence of clusters. Finally, draft genomes determined from these assemblies are presented in the last outer cycle as well as in the tree itself. and $9 \mathrm{Mb}$ for Burkholderia and Streptomyces, respectively). As an alternative approach we compared these draft genomes to the best matching reference genomes available using the besthit function implemented in RAST. Our analysis indicated that the two Streptomyces draft genomes (Mer-2-A and Mer-2-B) covered $81 \%$ and $83 \%$ of the gold standard genome Streptomyces avermitilis MA-4680, respectively. Similarly, although singlecopy gene analysis estimated only $45 \%$ and $36 \%$ completion for the two Burkholderia draft genomes, they covered $87-90 \%$ of the gold standard Burkholderia xenovorans LB400 chromosome 1.
These contrasting findings suggest that draft genome completion estimates based on single-copy gene families can underestimate the rate of recovery when multiple closely related bacterial genomes are present in the assembly.

The relative proportion of the reads associated with these genomes ranged between $<0.0001 \%$ and $>0.01 \%$ of the total reads sequenced from the original soil site [ $>12$ million reads available (Delmont et al., 2012)] while they represented 2-58\% of the reads recovered from microcosms after 4 months of treatment. Therefore, these genomes were enriched by several 


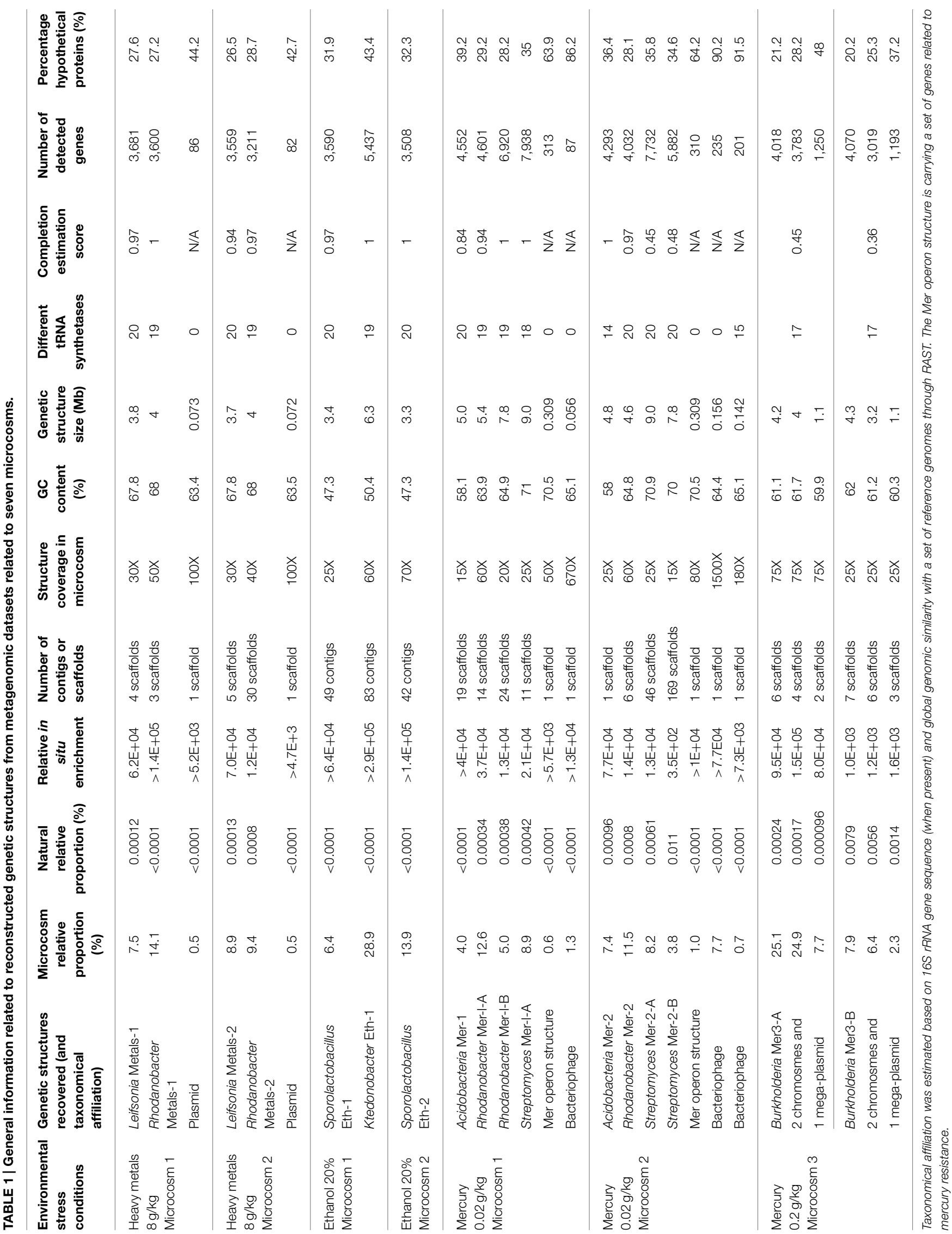


orders of magnitude during the incubation period, in contrast to the overall decline of total DNA observed after incubation. Genomes reconstructed from the ethanol enrichment condition were not detected in the natural community, with one of them related to the family Ktedonobacteraceae. Until now, this lineage had only one representative genome sequenced due to the difficulty cultivating this branch of the tree of life (Chang et al., 2011). This result emphasizes the interest of investigating rare members of the soil biosphere to recover the genomic content of novel taxonomical lineages. The recovery of this draft genome $(6.3 \mathrm{Mb})$ would have been impossible with canonical approaches that focus solely upon post-sequencing data treatment.

In addition, we observed three phage infections from the two microcosm replicates of mercury enrichment \#1, which were affiliated with the Streptomyces population based on the clustering tetranucleotide frequencies. Despite its small size of $156 \mathrm{~kb}$, the most dominant phage recruited $7.68 \%$ of all reads in the metagenomic data generated from the second microcosm representative of this ESC and reached 1,500× coverage, which was 38 times more frequent than the two dominant Streptomyces genomes reconstructed from the same microcosm. Functions for most genes carried by these three phages were unknown.
Overall, we recovered from one to four bacterial genomes, along with thousands of orphan genetic structures in each targeted microcosm, validating the strategy reconstructing a soil metagenome one ESC at the time.

\section{Different Mega-Plasmids Confer Mercury Resistance in Coexisting Burkholderia Populations}

Burkholderia populations often harbor a multi-replicon genome in nature (Chain et al., 2006). Here, we ordered and oriented scaffolds from the two Burkholderia genomes (mercury enrichment \#2) using $\mathrm{G}+\mathrm{C}$ skew and recovered two replicons and a megaplasmid for each of them (Figure 4). The observation of a well-defined $\mathrm{G}+\mathrm{C}$ skew provided an additional support for the reliability of our genomic reconstructions. A similar genomic organization was observed between the two Burkholderia genomes (e.g., tRNAs and flagellar genes operon in the replicon 1) and no differences could be detected between their full-length $16 \mathrm{~S}$ rRNA genes. On the other hand, most genes and functions were not shared between the two mega-plasmids (Figure S3). These mobile genetic elements also possessed a higher proportion of hypothetical proteins. Yet, mega-plasmids carried similar
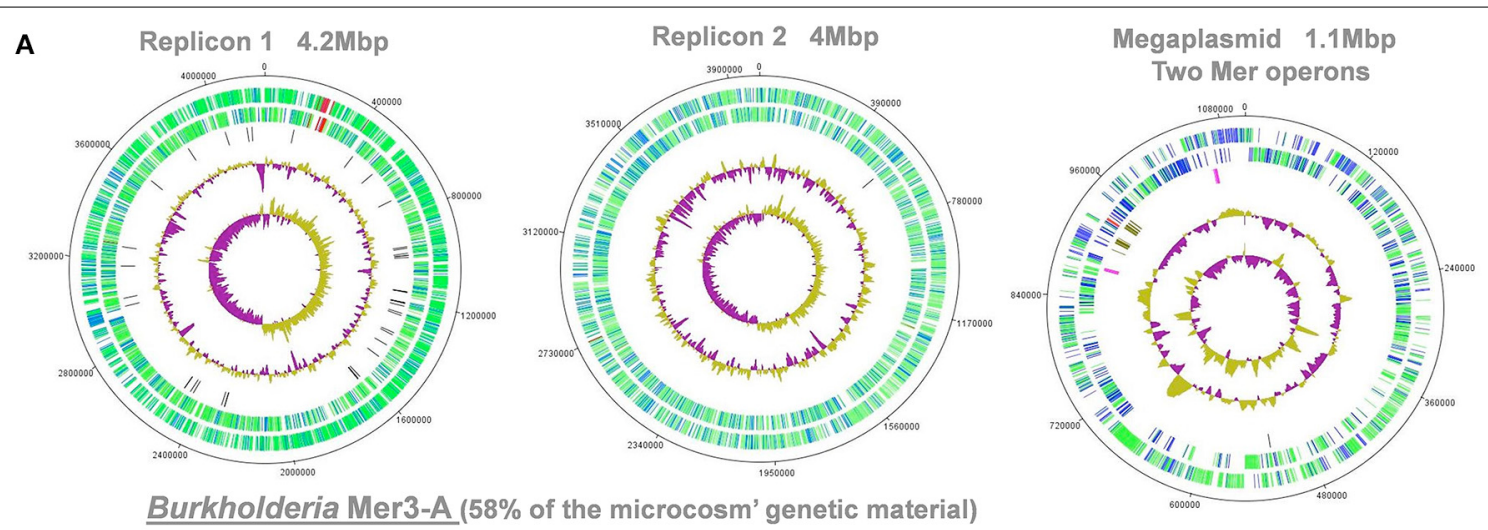

Burkholderia Mer3-A (58\% of the microcosm' genetic material)

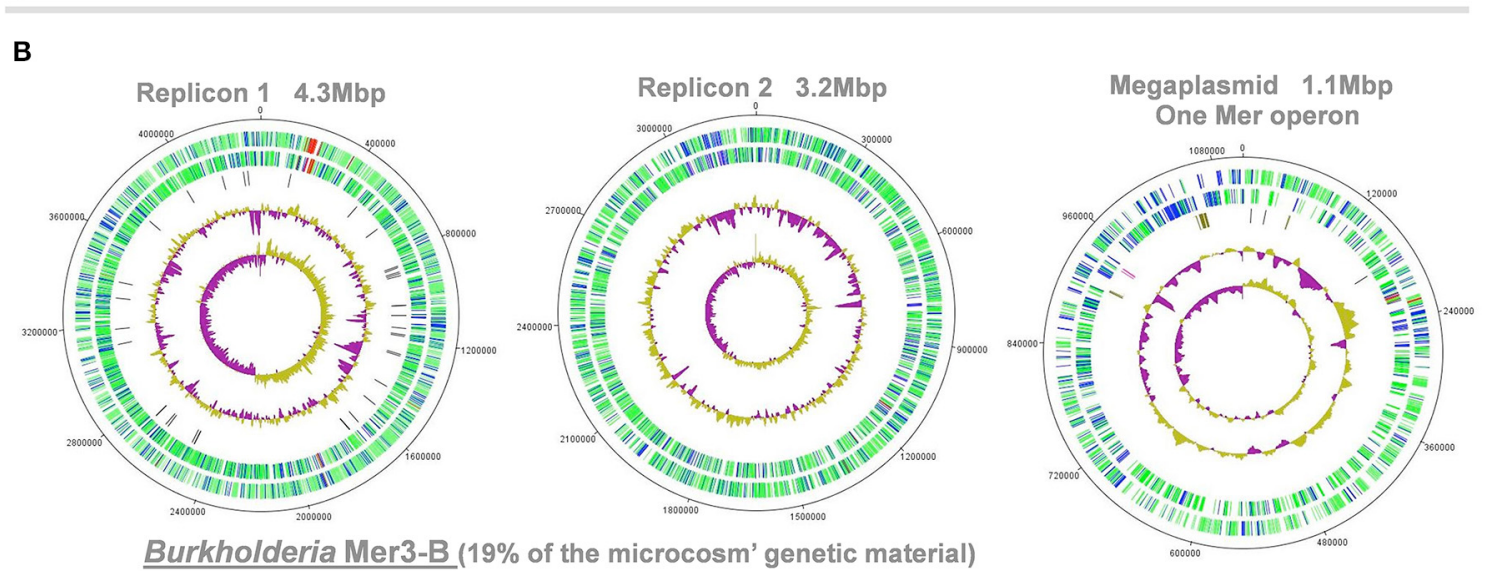

FIGURE 4 | Example of the two Burkholderia genomes reconstructed from the third replicate of mercury enrichment \#2. Panels $(A, B)$ represent replicons and mega-plasmid from Burkholderia Mer3-A and Mer3-B, respectively. Artemis and DNAPlotter were used to visualize the two replicons and mega-plasmid present in this microorganism. First (i.e., interior) and second circles represents GC skew and GC-content variations, respectively. Third circle represents the location of tRNAs (dark), conjugative (chestnut), and mercury (pink) related genes. Fourth and fifth circles represent genes of known (green) and unknown (blue) functions as well as genes related to flagellum (red) in the two possible frames. 
mercury resistance gene operons, suggesting a preponderant role in the ability of these coexisting Burkholderia populations to grow under the mercury ESC. Two mercury resistance operons found in Burkholderia Mer3-A (representing the most abundant population) further supports this suggestion. Similar operons have been found in plasmids worldwide (Barrineau et al., 1983; Osborn et al., 1997) while, to the best of our knowledge, not specifically in organisms affiliated to Burkholderia. However, these two populations may have acquired mercury resistance operons during the incubation period, and may not necessarily possess mercury resistance in the pristine soil.

\section{ESCs are not Redundant and Therefore Can Lead to a Large Genomic Catalogue from Soil}

Different taxonomic groups in our soil sample adapted to different ESCs. For instance, while ethanol stimulated the growth of Firmicutes, heavy metals stimulated Proteobacteria and Actinobacteria. We generated a tetranucleotide frequencybased tree to display the distribution of 8 taxonomical groups that were present in our microcosms (Figure 5). Recovered genomes for each ESC displayed near-identical distribution patterns across replicate microcosms, demonstrating high reproducibility of ESC treatments. In most cases genomes that were enriched under a particular ESC, were not detected in other ESCs. An exception to this was Rhodanobacter Metals-1, which was abundant in heavy metals enrichment \#2, and it was also detected in the mercury enrichment \#1 (another heavy metal) in lower abundance. Overall, each ESC provided a unique set of genomes, suggesting that more genomes could be recovered by applying additional conditions to this soil. The recovery of distinct organisms by only changing the concentration of mercury also suggests that a fine gradient of concentrations may stimulate the recovery of more genomes from organisms that prefer a specific range. It is indeed difficult to estimate what fraction of the soil microbial community that can be targeted from such enrichment approaches, yet ESCs may be useful to build a larger catalog of genes and their genomic contexts found in soil.

\section{Recovered Genomes Harbor Different Functional Pools Driven by Taxonomy}

We performed a network visualization using observed functions in recovered genomes from each ESC (Figure 6A). Similar genomes recovered from multiple microcosms lead to the identification of large number of core functions (e.g., about 3,500 identical genes for the two recovered Sporolactobacillus), as well as smaller, microcosm-specific ones (mostly hypothetical proteins and mobile elements). Micro-diversity traits are commonly found in cultivated strains of similar taxonomy, including in those displaying identical 16S rRNA genes (Jaspers and Overmann, 2004; Pena et al., 2010). Note that only the consensus assemblies of each genome were compared between replicates but that the genomic populations recovered from our study are likely to be polyclonal, contrasting with most micro-diversity analyses performed using isolate collections. We also generated an overall functional network using all 17 genomes and 11,299 functions they entailed (Figure 6B). The network analysis highlights the functional similarity of the five
Rhodanobacter genomes in spite of different ESCs they were found (combination of heavy metals vs. mercury alone). Each taxonomical group possessed unique functions (e.g., 2,088 and 2,545 functions only detected in Burkholderia and Streptomyces genomes, respectively).

\section{Genomic Functional Differences Suggest Distinct Ecological Roles within the Soil Microbiome}

Our genomic collection contained seven genera (Gram+/Gramratio was close to 1) with distinct functions. A majority of these organisms possessed gliding and flagellar motility capacities. Moreover, they harbored different metabolic capacities (e.g., Entner-Doudoroff pathway, betaglucoside utilization, fructose utilization) and strategies to target iron (e.g., ton and tol transport system, siderophore assembly) (Table 2). The Sporolactobacillus genomes possessed more than 40 genes related to spore formation, none of these genes being detected in the other genomes. Derived from the same condition and representing an unusual functional trait for Bacteria, the Ktedonobacter genome has the potential to produce both vitamin K1 (Phylloquinone) and K2 (Menaquinone) from Chorismate. The three Streptomyces genomes carried genes related to ethylmalonyl-CoA pathway of C2 assimilation, spore pigment biosynthetic cluster, sigmaB stress response regulation, sialic acid metabolism and siderophore assembly. As a potential industrial interest, several novel polyketide synthase modules were detected in these genomes. The two Burkholderia species possessed genes for degrading benzoate, but only one was carrying genes for a chloroaromatic degradation pathway, while the other harbored genes for degrading chlorobenzoate. When focusing on the nitrogen cycle, all these populations had the functional potential to assimilate ammonia and most of them could perform nitrite and nitrate ammonification. On the other hand, only the Sporolactobacillus genomes possessed adequate genetic information to fix nitrogen from the atmosphere (operon nifDKHENBQU). This is the first time this taxon is linked to nitrogen fixation, which is an important ecological trait in soil systems (Peoples et al., 1995; Bothe et al., 2006; Prosser et al., 2006). Finally, one Rhodanobacter genome possessed the genes required to perform denitrification. Rhodanobacter is involved in the soil nitrogen cycle through denitrification (Green et al., 2012; Kostka et al., 2012) and our metagenomic-derived genomes also harbored this functional trait.

\section{Enriched Populations Harbor Functional Traits Undetected in Culture Representatives}

We compared the genomes we recovered from our microcosms to genomes of cultivated organisms to identify novel functions. The five Rhodanobacter genomes recovered from our ESCs contained more than a thousand additional functions compared to the six culture-derived Rhodanobacter genomes currently available. These functions include secretion pathway proteins, membrane and outer membrane proteins, peptidases, a penicillin amidase, various transcriptional regulators, virulence proteins, chitin utilization and lignin degradation proteins as well as proteins related to cyanophycin metabolism (both cyanophycin synthase and cyanophycinase) and inositol catabolism. On the 

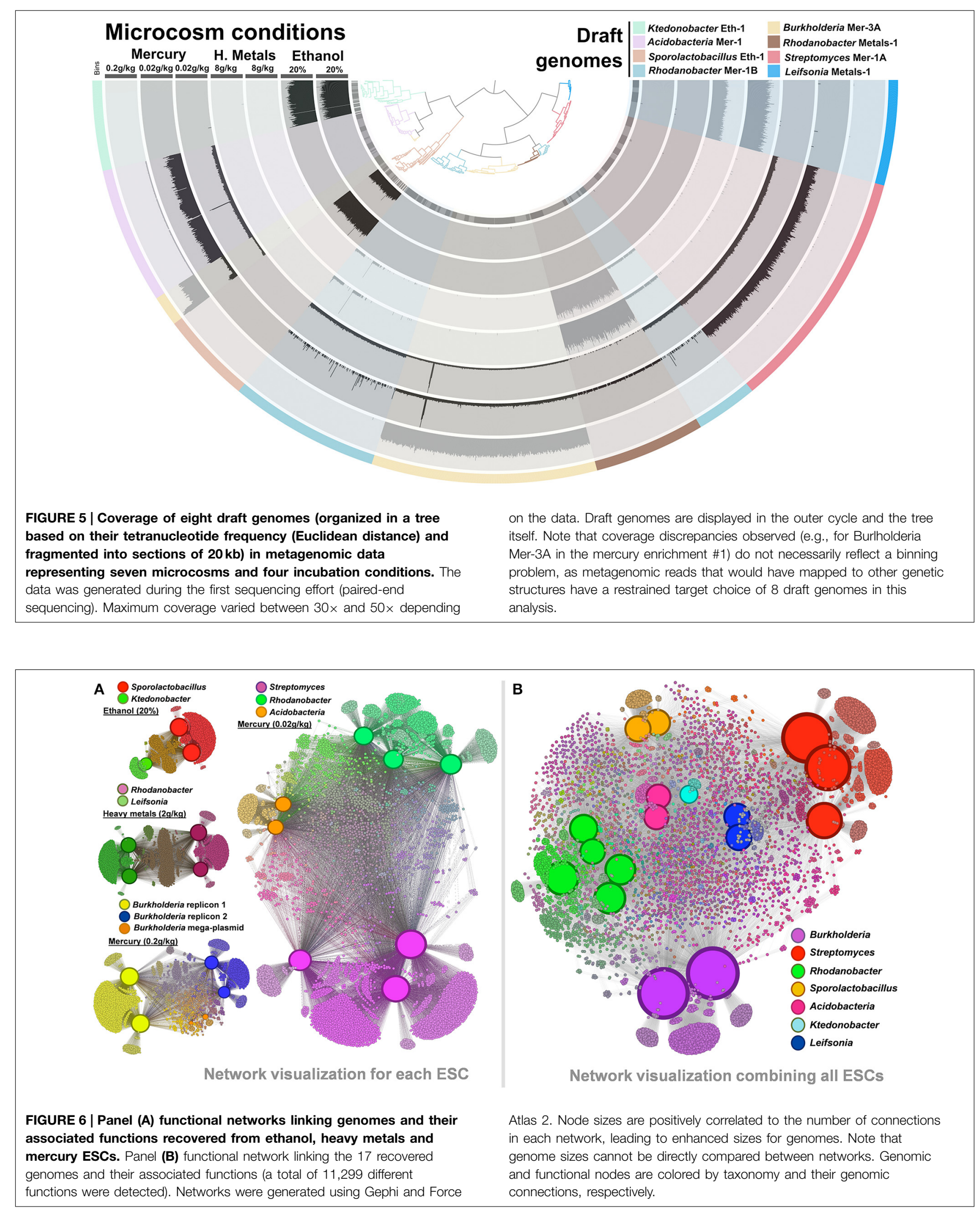


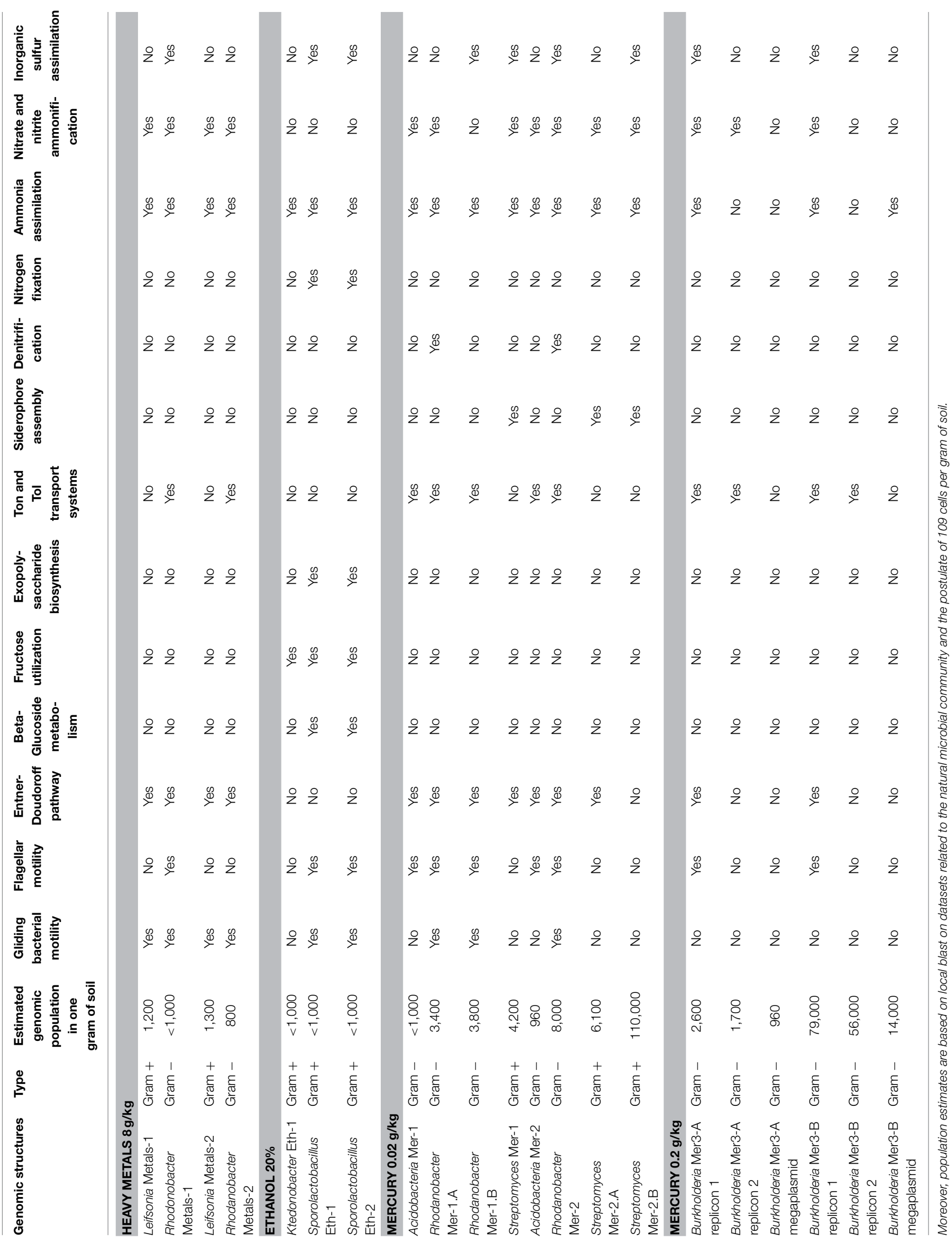




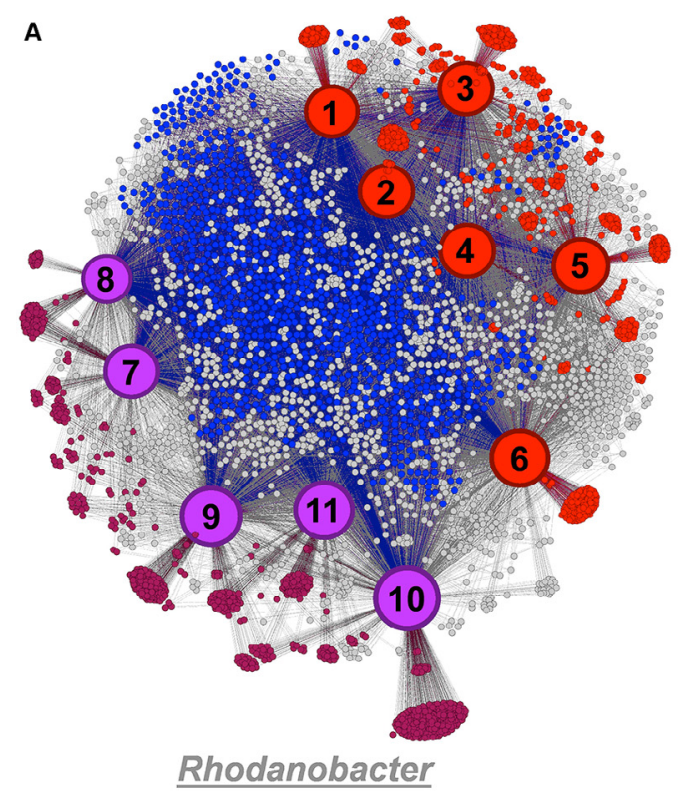

FIGURE 7 | Panel (A) functional network linking 11 Rhodanobacter genomes and their functionality (a total of 4,824 functions) using Gephi. Culture-derived genomes are represented by red nodes: $1, R$. spathiphylli B39; 2, R. fulvus Jip2; 3, R. sp. 115; 4, R. denitrificans 1162; 5, R. thiooxydans LCS2; 6, R. sp. 2APBS1. Metagenomic-derived genomes are represented by purple nodes: 7, R. Metals-1; 8, R. Metals-2; 9, R. Mer-1A; 10, R. Mer-1B; 11, R. Mer-2. Genomic node sizes are positively correlated to the number of connected functions. For the functional nodes, red and purple nodes represent functions detected only in one or more culture-derived or metagenomic-derived genome, respectively. Finally, blue functional nodes are detected in the 11 genomes. Panel (B) functional network linking 27 Streptomyces genomes and their functionality (a total of 11,032 functions) using Gephi.

Culture-derived genomes are represented by red nodes: 1, S. griseus

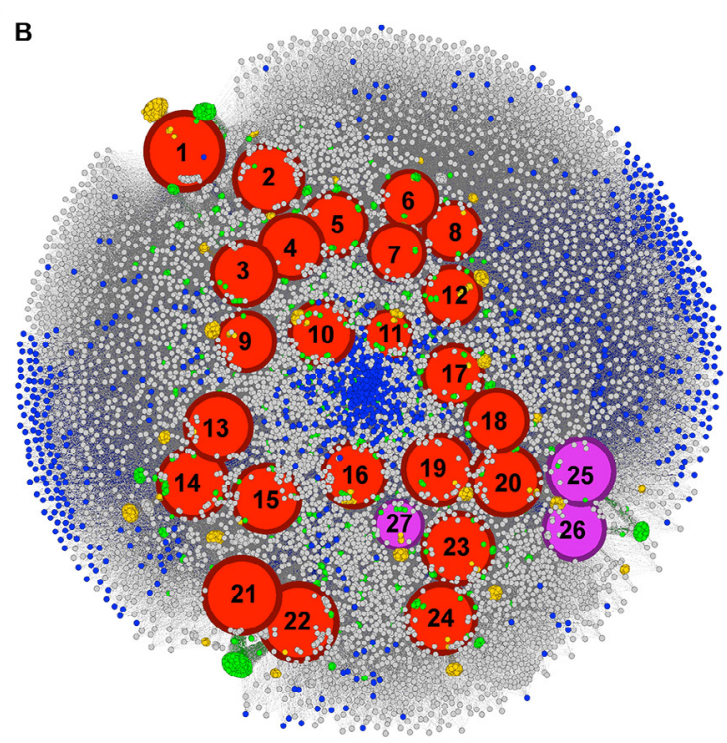

Streptomyces

NBRC 13350; 2, S. fulvissimus DSM 40593; 3, S. sp. Sirex AA- E; 4, S sp. PAM C26508; 5, S. flavogriseus ATCC 33331; 6, S. sp. GBA 94- 10; 7, S. sp. PVA 94- 07; 8, S. albus J1074; 9, S. sp. Tu6071; 10, S. venezuelae ATCC 10712; 11, S. cattleya DSM 46488; 12, S. clavuligerus ATCC 27064; 13, S. violaceusniger Tu 4113: 14, S. rapamycinicus NRRL 5491; 15, S. bingchenggensis BCW- 1; 16, S. ghanaensis ATCC 14672; 17, S. albulus CCRC 11814; 18, S. collinus Tu 365; 19, S. davawensis JCM 4913; 20, S. hygroscopicus 5008; 21, S. lividans 1326; 22, S. coelicolor A3(2); 23, S. scabiei 87.22; 24, S. avermitilis MA- 4680. Metagenomic-derived genomes are represented by purple nodes: $25, \mathrm{~S}$. Mer- 1A; 26, S. Mer- 2A; 27, S. Mer- 2B. Genomic node sizes are positively correlated to the number of connected functions. For the functional nodes, blues nodes are detected in all genomes and yellow and green nodes are detected in one and two genomes, respectively. other hand, 997 functions were detected in all Rhodanobacter genomes and 1159 only in the culture-derived genomes. The network analysis displayed separate groupings of cultivated and metagenomic analysis-based Rhodanobacter genomes, possibly due to differences in methodologies (Figure 7A). As another example, the three newly recovered Streptomyces genomes provided 330 functions undetected in the 24 culture-derived Streptomyces genomes, which represent 3\% increase of the 10,702 functions cataloged for this genus (Figure 7B). Novel functions included $\mathrm{ABC}$ transporters, Daunorubicin (a chemotherapy agent) resistance, a ferredoxin, four glycosyl hydrolase families, phenazine (an antibiotic) and pyochelin (a siderophore) biosynthesis proteins as well as pullulanase and neopullulanase (starch catabolism) genes. Streptomyces is a filamentous group of bacteria responsible for producing natural antibiotics that can be used in human and veterinary medicine (Malpartida and Hopwood, 1984). Antibiotic resistance in disease-causing bacteria is a growing concern (Thomson, 1999; Martinez and Baquero, 2000; Andersson and Hughes, 2010), and soil is a promising environment for new targets (Lin et al., 2015). Our recovery of Streptomyces draft genomes from multiple enrichment conditions suggests that enrichment studies could accelerate the discovery of novel bio-active compounds.

\section{Representation of Recovered Genetic Structures in Geographically Distinct Soil Biomes}

The 17 draft genomes we recovered from our ESCs represented about $0.03 \%$ of the natural Park Grass soil, based on metagenomic reads mapping at $97 \%$ identity cut-off. It is estimated that each gram of surface soil contains approximately $10^{9}$ bacterial cells, which suggests that the number of cells our genomes represent is up to 0.3 million per gram of this soil. We also traced the relative abundance of genomes we recovered in a metagenomic dataset of samples collected from distinct soil environments [cold and hot deserts, tropical and temperate forests, prairie, boreal forest, and tundra (Fierer et al., 2012)]. Although no genomes were recovered from these samples, Fierer et al. (2012) have reported different functional potentials based on short reads. Our recovered genomes recruited up to $0.1 \%$ and $0.2 \%$ of short reads from these samples at identity cut-offs of $97 \%$ and $90 \%$ (Figures 8A,B), suggesting that the taxonomic groups they represent are likely to occur globally. Moreover, genomes and orphan genetic structures (i.e., contigs 


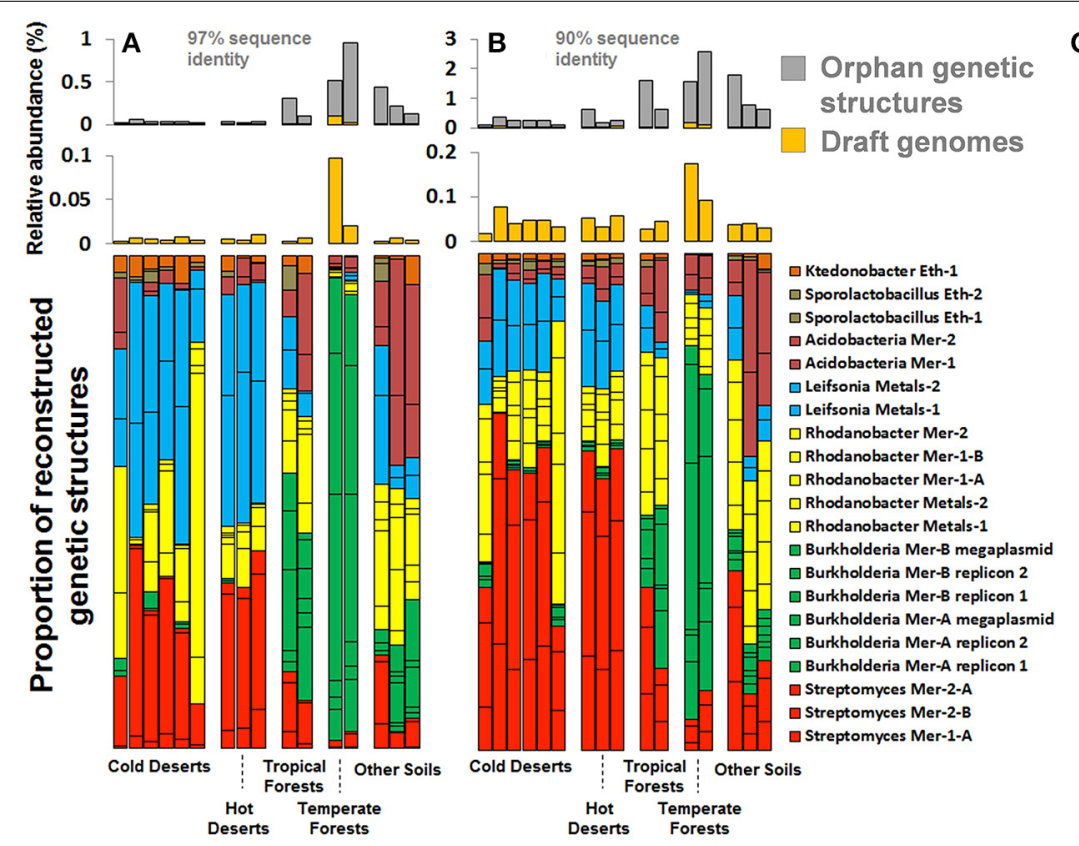

C

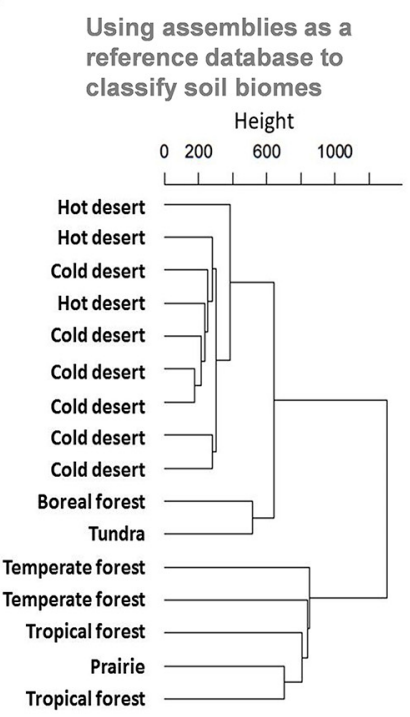

level. Panel (C) represents the classification of the same samples using our assemblies (genomes and orphan genetic structures) as a reference database and a $90 \%$ sequence identity cut-off for mapping. The dendrogram was generated using Ward's method with Euclidean distances. that were not binned into genomes) recovered from the Park Grass soil occurred in different proportion between biomes (e.g., Burkholderia more prevalent in temperate forests), providing clear geographic patterns for the recruited soil microbiome fraction. We subsequently used these genetic structures (more than half a million contigs averaging $1 \mathrm{~kb}$ in length) as a first soil reference database of its kind to classify soil biomes and defined tree groups depicting deserts, high latitude soils and temperate soils (Figure 8C). These observations only partly agree with results obtained using reference databases lacking soil reference genomes that required low stringency annotations (Fierer et al., 2012) and provide a first case study on how soil reference genomic databases can assist analyzing and partitioning samples collected from this environment.

\section{Conclusion}

Here we assembled $540 \mathrm{Mb}$ of genetic material (including 17 draft genomes) from a soil sample using enrichment strategies. The assembled genetic material constituted about $1 \%$ of the original soil microbiome, and we achieved this using about $10 \mathrm{~Gb}$ of sequencing and standard bioinformatics approaches. We detected these draft genomes also in soil samples collected from distant locations. Although the recovered genetic structures from Park Grass explain only $1 \%$ of the diversity, considering the absence of any genomes recovered from soil through metagenomics to date, our study demonstrates the efficacy of pre-sequencing enrichment applications as a way to break into the vastly unexplored soil microbiome.

\section{Acknowledgments}

We would like to thank Penny Hirsch and Ian Clark from the Rothamsted Research Center as well as Samuel Jacquiod from the Environmental Microbial Genomics group for providing soil samples. We also want to thank Joseph $\mathrm{H}$ Vineis for his precious assistance in bioinformatics and statistics. This research was supported by the French National Research Agency (Agence National de Recherche) project Metasoil (Projet ANR-08-GENM-025). TOD was funded by the Rhone-Alpes Région. $\mathrm{LM}$ was supported with a $\mathrm{PhD}$ fellowship from the Région Rhône-Alpes. No competing commercial interest by any of the authors was involved in this manuscript.

\section{Supplementary Material}

The Supplementary Material for this article can be found online at: http://journal.frontiersin.org/article/10.3389/fmicb. 2015.00358/abstract 


\section{References}

Albertsen, M., Hugenholtz, P., Skarshewski, A., Nielsen, K. L., Tyson, G. W., and Nielsen, P. H. (2013). Genome sequences of rare, uncultured bacteria obtained by differential coverage binning of multiple metagenomes. Nat. Biotechnol. 31, 533-538. doi: 10.1038/ nbt. 2579

Anantharaman, K., Breier, J. A., Sheik, C. S., and Dick, G. J. (2013). Evidence for hydrogen oxidation and metabolic plasticity in widespread deep-sea sulfur-oxidizing bacteria. Proc. Natl. Acad. Sci. U.S.A. 110, 330-335. doi: 10.1073/pnas. 1215340110

Andersson, D. I., and Hughes, D. (2010). Antibiotic resistance and its cost: is it possible to reverse resistance? Nat. Rev. Microbiol. 8, 260-271. doi: $10.1038 /$ nrmicro2319

Aziz, R. K., Bartels, D., Best, A. A., DeJongh, M., Disz, T., and Edwards, R. A., et al. (2008). The RAST Server: rapid annotations using subsystems technology. BMC genomics 9:75. doi: 10.1186/1471-2164-9-75

Barrineau, P., Gilbert, P., Jackson, W., Jones, C., Summers, A., and Wisdom, S. (1983). The DNA sequence of the mercury resistance operon of the IncFII plasmid NR1. J. Mol. Appl. Genet. 2, 601-619.

Bastian, M., Heymann, S., and Jacomy, M. (2009). Gephi: an open source software for exploring and manipulating networks. ICWSM 8, 361-362.

Bothe, H., Ferguson, S., and Newton, W. E. (2006). Biology of the Nitrogen Cycle. Amsterdam: Elsevier.

Boubakri, H., Beuf, M., Simonet, P., and Vogel, T. M. (2006). Development of metagenomic DNA shuffling for the construction of a xenobiotic gene. Gene 375, 87-94. doi: 10.1016/j.gene.2006.02.027

Carver, T., Thomson, N., Bleasby, A., Berriman, M., and Parkhill, J. (2009). DNAPlotter: circular and linear interactive genome visualization. Bioinformatics 25, 119-120. doi: 10.1093/bioinformatics/btn578

Chain, P. S., Denef, V. J., Konstantinidis, K. T., Vergez, L. M., Agulló L., and Reyes, V. L., et al. (2006). Burkholderia xenovorans LB400 harbors a multireplicon, 9.73-Mbp genome shaped for versatility. Proc. Natl. Acad. Sci. U.S.A. 103, 15280-15287. doi: 10.1073/pnas.0606924103

Chang, Y.-J., Land, M., Hauser, L., Chertkov, O., Del Rio, T. G., and Nolan, M., et al. (2011). Non-contiguous finished genome sequence and contextual data of the filamentous soil bacterium Ktedonobacter racemifer type strain (SOSP1-21T). Stand. Genomic Sci. 5, 97. doi: 10.4056/sigs.2114901

Cole, J. R., Wang, Q., Cardenas, E., Fish, J., Chai, B., and Farris, R. J., et al. (2009). The Ribosomal Database Project: improved alignments and new tools for rRNA analysis. Nucleic Acids Res. 37, D141-D145. doi: 10.1093/nar/ gkn879

Daniel, R. (2004). The soil metagenome-a rich resource for the discovery of novel natural products. Curr. Opin. Biotechnol. 15, 199-204. doi: 10.1016/j.copbio.2004.04.005

David, M. M., Cecillon, S., Warne, B. M., Prestat, E., Jansson, J. K., and Vogel, T. M. (2014). Microbial ecology of chlorinated solvent biodegradation. Environ. Microbiol. doi: 10.1111/1462-2920.12413. [Epub ahead of print].

Delmont, T. O., Francioli, D., Jacquesson, S., Laoudi, S., Mathieu, A., and Nesme, J., et al. (2014). Microbial community development and unseen diversity recovery in inoculated sterile soil. Biol. Fertil. Soils 50, 1069-1076. doi: 10.1007/s00374-0140925-8

Delmont, T. O., Malandain, C., Prestat, E., Larose, C., Monier, J. M., and Simonet, P., et al. (2011a). Metagenomic mining for microbiologists. ISME J. 5, 1837-1843. doi: 10.1038/ismej.2011.61

Delmont, T. O., Prestat, E., Keegan, K. P., Faubladier, M., Robe, P., and Clark, I. M., et al (2012). Structure, fluctuation and magnitude of a natural grassland soil metagenome. ISME J. 6, 1677-1687. doi: 10.1038/ismej.2011.197

Delmont, T. O., Robe, P., Cecillon, S., Clark, I. M., Constancias, F., and Simonet, P., et al. (2011b). Accessing the soil metagenome for studies of microbial diversity. Appl. Environ. Microbiol. 77, 1315-1324. doi: 10.1128/AEM.01526-10

Falkowski, P., Scholes, R., Boyle, E., Canadell, J., Canfield, D., and Elser, J., et al. (2000). The global carbon cycle: a test of our knowledge of earth as a system. Science 290, 291-296. doi: 10.1126/science.290.5490.291

Fierer, N., Leff, J. W., Adams, B. J., Nielsen, U. N., Bates, S. T., and Lauber, C. L., et al. (2012). Cross-biome metagenomic analyses of soil microbial communities and their functional attributes. Proc. Natl. Acad. Sci. U.S.A. 109, 21390-21395. doi: 10.1073/pnas.1215210110

Finzi, A. C., Austin, A. T., Cleland, E. E., Frey, S. D., Houlton, B. Z., and Wallenstein, M. D. (2011). Responses and feedbacks of coupled biogeochemical cycles to climate change: examples from terrestrial ecosystems. Front. Ecol. Environ. 9, 61-67. doi: 10.1890/100001

Galvão, T. C., Mohn, W. W., and de Lorenzo, V. (2005). Exploring the microbial biodegradation and biotransformation gene pool. Trends Biotechnol. 23, 497-506. doi: 10.1016/j.tibtech.2005.08.002

Gans, J., Wolinsky, M., and Dunbar, J. (2005). Computational improvements reveal great bacterial diversity and high metal toxicity in soil. Science 309, 1387-1390. doi: $10.1126 /$ science. 1112665

Garcia Martin, H., Ivanova, N., Kunin, V., Warnecke, F., Barry, K. W., and McHardy, A. C., et al. (2006). Metagenomic analysis of two enhanced biological phosphorus removal (EBPR) sludge communities. Nat. Biotechnol. 24, 1263-1269. doi: 10.1038/nbt1247

Giller, K., Beare, M., Lavelle, P., Izac, A., and Swift, M. (1997). Agricultural intensification, soil biodiversity and agroecosystem function. Appl. Soil Ecol. 6, 3-16. doi: 10.1016/S0929-1393(96)00149-7

Green, S. J., Prakash, O., Jasrotia, P., Overholt, W. A., Cardenas, E., and Hubbard, D., et al. (2012). Denitrifying bacteria from the genus Rhodanobacter dominate bacterial communities in the highly contaminated subsurface of a nuclear legacy waste site. Appl. Environ. Microbiol. 78, 1039-1047. doi: 10.1128/AEM.06435-11

Handelsman, J., Rondon, M. R., Brady, S. F., Clardy, J., and Goodman, R. M. (1998). Molecular biological access to the chemistry of unknown soil microbes: a new frontier for natural products. Chem. Biol. 5, R245-249. doi: 10.1016/S1074-5521(98)90108-9

Hess, M., Sczyrba, A., Egan, R., Kim, T. W., Chokhawala, H., and Schroth, G., et al. (2011). Metagenomic discovery of biomass-degrading genes and genomes from cow rumen. Science 331, 463-467. doi: 10.1126/science.1200387

Howe, A. C., Jansson, J. K., Malfatti, S. A., Tringe, S. G., Tiedje, J. M., and Brown, C. T. (2014). Tackling soil diversity with the assembly of large, complex metagenomes. Proc. Natl. Acad. Sci. U.S.A. 111, 4904-4909. doi: 10.1073/pnas.1402564111

Ibekwe, A., Papiernik, S., Gan, J., Yates, S., Crowley, D., and Yang, C. H. (2001). Microcosm enrichment of 1,3-dichloropropene-degrading soil microbial communities in a compost-amended soil. J. Appl. Microbiol. 91, 668-676. doi: 10.1046/j.1365-2672.2001.01431.x

Jacquiod, S., Franqueville, L., Cécillon, S., Vogel, T. M., and Simonet, P. (2013). Soil bacterial community shifts after chitin enrichment: an integrative metagenomic approach. PLoS ONE 8:e79699. doi: 10.1371/journal.pone.0079699

Jaspers, E., and Overmann, J. (2004). Ecological significance of microdiversity: identical $16 \mathrm{~S}$ rRNA gene sequences can be found in bacteria with highly divergent genomes and ecophysiologies. Appl. Environ. Microbiol. 70, 4831-4839. doi: 10.1128/AEM.70.8.4831-4839.2004

Kennedy, A., and Smith, K. (1995). Soil microbial diversity and the sustainability of agricultural soils. Plant Soil 170, 75-86. doi: 10.1007/BF02183056

Kostka, J. E., Green, S. J., Rishishwar, L., Prakash, O., Katz, L. S., and MariñoRamírez, L., et al (2012). Genome sequences for six Rhodanobacter strains, isolated from soils and the terrestrial subsurface, with variable denitrification capabilities. J. Bacteriol. 194, 4461-4462. doi: 10.1128/JB.00871-12

Lin, L. L., Schneider, T., Peoples, A. J., Spoering, A. L., Engels, I., and Conlon, B. P., et al (2015). A new antibiotic kills pathogens without detectable resistance. Nature 517, 455-459. doi: 10.1038/nature14098

Liu, Z., Klatt, C. G., Ludwig, M., Rusch, D. B., Jensen, S. I., and Kühl, M., et al (2012). 'Candidatus Thermochlorobacter aerophilum:'an aerobic chlorophotoheterotrophic member of the phylum Chlorobi defined by metagenomics and metatranscriptomics. ISME J. 6, 1869-1882. doi: 10.1038/ismej.2012.24

Luo, C., Tsementzi, D., Kyrpides, N. C., and Konstantinidis, K. T. (2012). Individual genome assembly from complex community short-read metagenomic datasets. ISME J. 6, 898-901. doi: 10.1038/ismej.2011.147

Mackelprang, R., Waldrop, M. P., DeAngelis, K. M., David, M. M., Chavarria, K. L., and Blazewicz, S. J., et al (2011). Metagenomic analysis of a permafrost microbial community reveals a rapid response to thaw. Nature 480, 368-371. doi: $10.1038 /$ nature10576 
Malpartida, F., and Hopwood, D. (1984). Molecular cloning of the whole biosynthetic pathway of a Streptomyces antibiotic and its expression in a heterologous host. Nature 309, 462-464.

Martinez, J., and Baquero, F. (2000). Mutation frequencies and antibiotic resistance. Antimicrob. Agents Chemother. 44, 1771-1777. doi: 10.1128/AAC.44.7.1771-1777.2000

McIlroy, S. J., Albertsen, M., Andresen, E. K., Saunders, A. M., Kristiansen, R., and Stokholm-Bjerregaard, M., et al. (2013). 'Candidatus Competibacter'-lineage genomes retrieved from metagenomes reveal functional metabolic diversity. ISME J. 8, 613-624. doi: 10.1038/ismej.2013.162

McKew, B. A., Coulon, F., Osborn, A. M., Timmis, K. N., and McGenity, T. J. (2007). Determining the identity and roles of oil-metabolizing marine bacteria from the Thames Estuary, UK. Environ. Microbiol. 9, 165-176. doi: 10.1111/j.1462-2920.2006.01125.x

Mende, D. R., Waller, A. S., Sunagawa, S., Järvelin, A. I., Chan, M. M., and Arumugam, M., et al. (2012). Assessment of metagenomic assembly using simulated next generation sequencing data. PLoS ONE 7:e31386. doi: 10.1371/journal.pone.0031386

Meyer, F., Paarmann, D., D’Souza, M., Olson, R., Glass, E. M., and Kubal, M., et al. (2008). The metagenomics RAST server - a public resource for the automatic phylogenetic and functional analysis of metagenomes. BMC Bioinformatics 9:386. doi: 10.1186/1471-2105-9-386

Narasingarao, P., Podell, S., Ugalde, J. A., Brochier-Armanet, C., Emerson, J. B., and Brocks, J. J., et al. (2012). De novo metagenomic assembly reveals abundant novel major lineage of Archaea in hypersaline microbial communities. ISME J. 6, 81-93. doi: 10.1038/ismej.2011.78

Nesme, J., Cécillon, S., Delmont, T. O., Monier, J.-M., Vogel, T. M., and Simonet, P. (2014). Large-scale metagenomic-based study of antibiotic resistance in the environment. Curr. Biol. 24, 1096-1100. doi: 10.1016/j.cub.2014.03.036

Nielsen, H. B., Almeida, M., Juncker, A. S., Rasmussen, S., Li, J., and Sunagawa, S., et al. (2014). Identification and assembly of genomes and genetic elements in complex metagenomic samples without using reference genomes. Nat. Biotechnol. 32, 822-828. doi: 10.1038/nbt.2939

Oksanen, J., Kindt, R., Legendre, P., O’Hara, B., Stevens, M. H. H., Oksanen, M. J., et al. (2007). The Vegan Package. Community ecology package.

Osborn, A. M., Bruce, K. D., Strike, P., and Ritchie, D. A. (1997). Distribution, diversity and evolution of the bacterial mercury resistance (mer) operon. FEMS Microbiol. Rev. 19, 239-262. doi: 10.1111/j.1574-6976.1997.tb00300.x

Pell, J., Hintze, A., Canino-Koning, R., Howe, A., Tiedje, J. M., and Brown, C. T. (2012). Scaling metagenome sequence assembly with probabilistic de Bruijn graphs. Proc. Natl. Acad. Sci. U.S.A. 109, 13272-13277. doi: 10.1073/pnas.1121464109

Pena, A., Teeling, H., Huerta-Cepas, J., Santos, F., Yarza, P., and Brito-Echeverría, J., et al. (2010). Fine-scale evolution: genomic, phenotypic and ecological differentiation in two coexisting Salinibacter ruber strains. ISME J. 4, 882-895. doi: 10.1038/ismej.2010.6

Peoples, M., Herridge, D., and Ladha, J. (1995). Biological nitrogen fixation: an efficient source of nitrogen for sustainable agricultural production? Plant Soil 174, 3-28. doi: 10.1007/BF00032239

Prosser, J. I., Rangel-Castro, J. I., and Killham, K. (2006). Studying plant-microbe interactions using stable isotope technologies. Curr. Opin. Biotechnol. 17, 98-102. doi: 10.1016/j.copbio.2006.01.001

Roesch, L. F., Fulthorpe, R. R., Riva, A., Casella, G., Hadwin, A. K., and Kent, A. D., et al. (2007). Pyrosequencing enumerates and contrasts soil microbial diversity. ISME J. 1, 283-290. doi: 10.1038/ismej.2007.53
Rondon, M. R., August, P. R., Bettermann, A. D., Brady, S. F., Grossman, T. H., and Liles, M. R., et al. (2000). Cloning the soil metagenome: a strategy for accessing the genetic and functional diversity of uncultured microorganisms. Appl. Environ. Microbiol. 66, 2541-2547. doi: 10.1128/AEM.66.6.25412547.2000

Sharon, I., Morowitz, M. J., Thomas, B. C., Costello, E. K., Relman, D. A., and Banfield, J. F. (2013). Time series community genomics analysis reveals rapid shifts in bacterial species, strains, and phage during infant gut colonization. Genome Res. 23, 111-120. doi: 10.1101/gr.142315.112

Stahl, D. A., Lane, D. J., Olsen, G. J., and Pace, N. R. (1984). Analysis of hydrothermal vent-associated symbionts by ribosomal RNA sequences. Science 224: 409-411. doi: 10.1126/science.224.4647.409

Thomson, C. J. (1999). The global epidemiology of resistance to ciprofloxacin and the changing nature of antibiotic resistance: a 10 year perspective. J. Antimicrob. Chemother. 43, 31-40. doi: 10.1093/jac/43.suppl_1.31

Torsvik, V., Ovreas, L., and Thingstad, T. F. (2002). Prokaryotic diversitymagnitude, dynamics, and controlling factors. Science 296, 1064-1066. doi: $10.1126 /$ science. 1071698

Tringe, S. G., von Mering, C., Kobayashi, A., Salamov, A. A., Chen, K., and Chang, H. W., et al. (2005). Comparative metagenomics of microbial communities. Science 308, 554-557. doi: 10.1126/science.1107851

Tyson, G. W., Chapman, J., Hugenholtz, P., Allen, E. E., Ram, R. J., and Richardson, P. M., et al. (2004). Community structure and metabolism through reconstruction of microbial genomes from the environment. Nature 428, 37-43. doi: $10.1038 /$ nature 02340

Vogel, T. M., Simonet, P., Jansson, J. K., Hirsch, P. R., Tiedje, J. M., and Van Elsas, J. D., et al. (2009). TerraGenome: a consortium for the sequencing of a soil metagenome. Nat. Rev. Microbiol. 7, 252. doi: 10.1038/ nrmicro2119

Wagner-Döbler, I., Bennasar, A., Vancanneyt, M., Strömpl, C., Brümmer, I., and Eichner, C., et al. (1998). Microcosm enrichment of biphenyl-degrading microbial communities from soils and sediments. Appl. Environ. Microbiol. 64, 3014-3022.

Wommack, K. E., Bhavsar, J., and Ravel, J. (2008). Metagenomics: read length matters. Appl. Environ. Microbiol. 74, 1453-1463. doi: 10.1128/AEM.02181-07

Wu, M., and Eisen, J. A. (2008). A simple, fast, and accurate method of phylogenomic inference. Genome Biol. 9:R151. doi: 10.1186/gb-2008-9-10r151

Yakimov, M. M., Denaro, R., Genovese, M., Cappello, S., D’Auria, G., and Chernikova, T. N., et al. (2005). Natural microbial diversity in superficial sediments of Milazzo Harbor (Sicily) and community successions during microcosm enrichment with various hydrocarbons. Environ. Microbiol. 7, 1426-1441. doi: 10.1111/j.1462-5822.2005.00829.x

Conflict of Interest Statement: The authors declare that the research was conducted in the absence of any commercial or financial relationships that could be construed as a potential conflict of interest.

Copyright (C) 2015 Delmont, Eren, Maccario, Prestat, Esen, Pelletier, Le Paslier, Simonet and Vogel. This is an open-access article distributed under the terms of the Creative Commons Attribution License (CC BY). The use, distribution or reproduction in other forums is permitted, provided the original author(s) or licensor are credited and that the original publication in this journal is cited, in accordance with accepted academic practice. No use, distribution or reproduction is permitted which does not comply with these terms. 